\title{
EWPD constraints on flavor symmetric vector fields
}

\author{
Benjamín Grinstein, ${ }^{a}$ Christopher W. Murphy ${ }^{a}$ and Michael Trott $^{b}$ \\ ${ }^{a}$ Department of Physics, University of California, \\ San Diego, La Jolla, CA 92093 USA \\ ${ }^{b}$ Theory Division, Physics Department, CERN, \\ CH-1211 Geneva 23, Switzerland \\ E-mail: bgrinstein@ucsd.edu, cmurphy@physics.ucsd.edu, \\ michael.trott@cern.ch
}

ABSTRACT: Electroweak precision data constraints on flavor symmetric vector fields are determined. The flavor multiplets of spin one that we examine are the complete set of fields that couple to quark bi-linears at tree level while not initially breaking the quark global flavor symmetry group. Flavor safe vector masses proximate to, and in some cases below, the electroweak symmetry breaking scale are found to be allowed. Many of these fields provide a flavor safe mechanism to explain the $t \bar{t}$ forward backward anomaly, and can simultaneously significantly raise the allowed values of the Standard Model Higgs mass consistent with electroweak precision data.

KeYwords: Phenomenological Models

ARXIV EPRINT: 1110.5361 


\section{Contents}

1 Introduction 1

2 Electroweak sector of MFV vector Lagrangians 3

2.1 Cases V, VI, and VIII 4

2.2 Case VII 5

2.3 Case IX 6

2.4 Cases X and XI 7

2.5 Counterterms and higher-dimensional operators 8

3 Fit to electroweak precision data $\quad 9$

$\begin{array}{llr}4 & \text { Results } & 10\end{array}$

4.1 Cases V, VI, and VIII 11

4.2 Case VII 12

$\begin{array}{lll}4.3 & \text { Case IX } & 12\end{array}$

$\begin{array}{ll}4.4 \text { Cases X and XI } & 12\end{array}$

$\begin{array}{lll}5 & \text { Conclusions } & 13\end{array}$

$\begin{array}{ll}\text { A Self-energy form factors } & 16\end{array}$

\section{Introduction}

The global flavor symmetry group of the Standard Model (SM) is only broken by the Yukawa matrices. As a result, the SM predicts a non-trivial and definite pattern in flavor changing decays and meson mixing observables; there are no flavor changing neutral currents (FCNC's) at tree level, and flavor changing charged currents follow the pattern of the CKM matrix. The predicted pattern of flavor changing observables in the SM is consistent with what is observed in precise measurements of $\operatorname{Br}(b \rightarrow s+\gamma), K^{0}-\bar{K}^{0}, B^{0}-\bar{B}^{0}$ mixing, etc. The lack of any apparent pattern of statistically significant deviations in these observables $^{1}$ places strong constraints on New Physics (NP) at the TeV scale invoked to resolve the hierarchy problem of the electroweak symmetry breaking (EWSB) scale, $v$, in the SM.

One way to attempt to reconcile these facts with NP that appears at the $\sim \mathrm{TeV}$ scale is to assume that the NP has a non-generic flavor structure. A popular assumption that can frequently accommodate this tension is to assume that NP has exactly the dominant flavor breaking pattern that has been experimentally established, i.e., that NP that couples to

\footnotetext{
${ }^{1}$ Note that some sets of measurements do show interesting patterns of deviations from the SM, in particular the measurement of the CP violating phase in $B_{s}$ mixing at the Tevatron through dimuon final states and $B \rightarrow J / \psi \phi$; see $[1,2]$ for related discussions. The recently reported $L_{H C} C_{\mathrm{b}}$ measurement [3] of $B \rightarrow J / \psi \phi$ does not support a NP interpretation of this data, while recent updates to D $\varnothing$ measurements of the same decay [4] are still able to accommodate the large phase required by the dimuon anomaly.
} 
quarks has the same flavor breaking structure as in the SM. This is known as the principle of Minimal Flavor Violation (MFV), which states that there is a unique source of flavor symmetry breaking [5-7] at scales measurable in flavor physics observables. Another, closely related assumption is that a $\mathrm{TeV}$ scale new physics sector is completely flavor symmetric with respect to the quark flavor symmetry group $-\mathrm{G}_{\mathrm{F}}=\mathrm{U}(3)_{\mathrm{Q}} \times \mathrm{U}(3)_{\mathrm{U}} \times$ $\mathrm{U}(3)_{\mathrm{D}}$. Breaking of this flavor symmetry is not completely absent because the quark masses already break $\mathrm{G}_{\mathrm{F}}$ through Yukawa interactions

$$
\mathscr{L}_{Y}=Y_{U} \bar{u}_{R} H^{T} i \sigma_{2} Q_{L}-Y_{D} \bar{d}_{R} H^{\dagger} Q_{L}+\text { h.c. }
$$

and this breaking will appear in loop corrections to the couplings of the NP to quark bilinears. This flavor breaking however will follow the SM pattern and be of an MFV form.

Further flavor breaking can be MFV like or deviate from the pattern expected in MFV. The top quark is the only quark with an $\mathcal{O}(1)$ Yukawa coupling, $y_{t}$. This large coupling generally forces phenomenologically viable $\sim v$ scale NP flavor breaking effects $\propto y_{t}$ to be aligned with the SM flavor breaking. This can be taken to mean that flavor breaking involving the third generation $\left(\mathrm{G}_{\mathrm{F}} \rightarrow \mathrm{H}_{\mathrm{F}}\right.$ where $\left.\mathrm{H}_{\mathrm{F}}=\mathrm{U}(2)_{\mathrm{Q}} \times \mathrm{U}(2)_{\mathrm{U}} \times \mathrm{U}(2)_{\mathrm{D}} \times \mathrm{U}(1)^{3}\right)$ should be aligned with the SM. Versions of this scenario are referred to as next to minimal flavor violation in the literature [8].

The scalar and vector fields that can couple to the SM at dimension four respecting SM gauge symmetry and not breaking $G_{F}$ have recently been classified and studied in some detail [9-11]. In this paper, we examine oblique electroweak precision data (EWPD) corrections due to the flavor symmetric vector field multiplets of this form in much more detail. We seek to perform as general an analysis of EWPD as possible on phenomenologically interesting flavor symmetric vector fields in this paper, and consequently only consider the (large) $\mathrm{G}_{\mathrm{F}} \rightarrow \mathrm{H}_{\mathrm{F}}$ flavor breaking in the mass spectrum. Sub-dominant flavor breaking could be MFV-like - where all flavor breaking comes with SM Yukawa insertions - or could deviate from the SM pattern of flavor breaking. In the later case, potentially important flavor changing observable based constraints could exist. For oblique EWPD constraints, only the leading flavor breaking we include is expected to be relevant. In fact, we will show that the breaking of $\mathrm{G}_{\mathrm{F}}$ is not directly related to the breaking of custodial symmetry $\mathrm{SU}_{\mathrm{C}}(2)$ to the approximation we work to, and thus we can neglect flavor breaking in general when considering oblique EWPD constraints. We find that vector masses $m_{v} \sim v$ are consistent with EWPD in most of the allowed representations, and in certain cases in large regions of parameter space. The allowed vector representations [11] are shown in table 1.

Note that interest in flavor symmetric fields has been increased recently due to the ability of a number of these representations to explain the CDF and DØ measurements [12, 13] of an anomalously large $t \bar{t}$ forward backward asymmetry, $A_{\mathrm{FB}}^{t \bar{t}}$. Flavor symmetric fields can in principle explain this anomaly in a flavor safe manner [14]. In addition, some fields of this form can potentially explain both the $t \bar{t}$ anomaly and same sign dimuon anomaly reported in [15] by $\mathrm{D} \varnothing$ at the same time [11]. ${ }^{2}$

\footnotetext{
${ }^{2}$ However, it should be noted that recent LHC results on the $t \bar{t}$ invariant mass spectrum [16] and the measurement of time dependent $B \rightarrow J / \psi \phi$ have cast some doubt on the NP interpretation of Tevatron anomalies in $A_{\mathrm{FB}}^{t \bar{t}}$ or the dimuon anomaly [3].
} 


\begin{tabular}{|c|c|c|c|c|c|}
\hline Case & $\mathrm{SU}(3)_{\mathrm{c}}$ & $\mathrm{SU}_{\mathrm{L}}(2)$ & $\mathrm{U}(1)_{\mathrm{Y}}$ & $\mathrm{SU}(3)_{\mathrm{U}_{\mathrm{R}}} \times \mathrm{SU}(3)_{\mathrm{D}_{\mathrm{R}}} \times \mathrm{SU}(3)_{\mathrm{Q}_{\mathrm{L}}}$ & couples to \\
\hline $\mathrm{I}_{\mathrm{s}, \mathrm{o}}$ & 1,8 & 1 & 0 & $(1,1,1)$ & $\bar{d}_{R} \gamma^{\mu} d_{R}$ \\
$\mathrm{II}_{\mathrm{s}, \mathrm{o}}$ & 1,8 & 1 & 0 & $(1,1,1)$ & $\bar{u}_{R} \gamma^{\mu} u_{R}$ \\
$\mathrm{III}_{\mathrm{s}, \mathrm{o}}$ & 1,8 & 1 & 0 & $(1,1,1)$ & $\bar{Q}_{L} \gamma^{\mu} Q_{L}$ \\
$\mathrm{IV}_{\mathrm{s}, \mathrm{o}}$ & 1,8 & 3 & 0 & $(1,1,1)$ & $\bar{Q}_{L} \gamma^{\mu} Q_{L}$ \\
\hline $\mathrm{V}_{\mathrm{s}, \mathrm{o}}$ & 1,8 & 1 & 0 & $(1,8,1)$ & $\bar{d}_{R} \gamma^{\mu} d_{R}$ \\
$\mathrm{VI}_{\mathrm{s}, \mathrm{o}}$ & 1,8 & 1 & 0 & $(8,1,1)$ & $\bar{u}_{R} \gamma^{\mu} u_{R}$ \\
$\mathrm{VII}_{\mathrm{s}, \mathrm{o}}$ & 1,8 & 1 & -1 & $(\overline{3}, 3,1)$ & $\bar{d}_{R} \gamma^{\mu} u_{R}$ \\
$\mathrm{VIII}_{\mathrm{s}, \mathrm{o}}$ & 1,8 & 1 & 0 & $(1,1,8)$ & $\bar{Q}_{L} \gamma^{\mu} Q_{L}$ \\
$\mathrm{IX}_{\mathrm{s}, \mathrm{o}}$ & 1,8 & 3 & 0 & $(1,1,8)$ & $\bar{Q}_{L} \gamma^{\mu} Q_{L}$ \\
$\mathrm{X}_{\overline{3}, 6}$ & $\overline{3}, 6$ & 2 & $-1 / 6$ & $(1,3,3)$ & $\bar{d}_{R} \gamma^{\mu} Q_{R}$ \\
$\mathrm{XI}_{\overline{3}, 6}$ & $\overline{3}, 6$ & 2 & $5 / 6$ & $(3,1,3)$ & $\bar{u}_{R} \gamma^{\mu} Q_{R}$ \\
\hline
\end{tabular}

Table 1. $\mathrm{G}_{\mathrm{F}}$ symmetric vector representations from ref. [11].

EWPD constrains the vector fields we study through effectively constraining the higher dimensional operators that are present in the theory when the NP is integrated out. Oblique EWPD constraints can generally be studied by using the STU parameters [17-19] which assumes that the masses of the new states are $\sim \mathrm{TeV}$. When considering masses comparable to (or lower than) the EWSB scale, the STUWVX formalism of [20] which does not expand in $v^{2} / m_{\mathrm{NP}}^{2}$ is preferred and can be more constraining [23]. The latter formalism reduces to the STU parameters when large NP scales are present and we will use the STUWVX formalism of [20] in this paper, as we are interesting in studying how light the vector multiplet masses can be.

\section{Electroweak sector of MFV vector Lagrangians}

Table 1 lists the $\mathrm{G}_{\mathrm{F}}$ symmetric representations that couple to quark bilinears without Yukawa suppression, while preserving SM gauge invariance. ${ }^{3}$ In this section, we construct the gauge sector of these Lagrangians ${ }^{4}$ for cases V-XI and determine the contribution of these fields to the self energies of the SM gauge bosons. As massive flavor symmetric vectors are effective fields in a non-renormalizable extension of the SM, we also consider the contribution of higher-dimension operators, which are suppressed by the cutoff scale, $\Lambda$, of the effective theory [22]. These operators are necessary to obtain finite oblique corrections in some cases. One naively expects the scaling $\Lambda \sim 4 \pi m_{v}$, but the separation of these scales can be smaller. Indeed, this might be expected to be the case due to the relatively large number of degrees of freedom in the vector multiplets. As a non-perturbative study is clearly beyond the scope of this initial work, we assume that the operators suppressed by higher powers of $\Lambda$ that we do not retain are sufficiently suppressed.

\footnotetext{
${ }^{3}$ Cases I-IV are flavor singlets and are already discussed extensively in the literature. See ref. [21] for a recent discussion.

${ }^{4}$ See [11] for the Yukawa sectors of these models and related phenomenological constraints.
} 


\subsection{Cases V, VI, and VIII}

These cases are not charged under the electroweak gauge group. The vector fields are parameterized using Gell-Mann matrices for the color, $\tau^{A}$, and the flavor, $t^{B}$, representations. The Lagrangians in the mass eigenstate basis for cases $C=\{V, V I, V I I I\}$ are

$$
\begin{aligned}
\mathscr{L}_{C} & =\left(1+\delta_{a, o}\right)\left(-\mathscr{L}_{C}^{\text {kin }}+\mathscr{L}_{C}^{\text {mass }}\right)+\mathscr{L}_{C}^{\text {int }}+\mathscr{L}_{C}^{\text {Yuk }}+\text { h.c. }, \\
\mathscr{L}_{C}^{\text {kin }} & =\frac{1}{2} \operatorname{Tr}\left(V^{a ; \mu \nu} V_{\mu \nu}^{a ; \dagger}\right)+\frac{\kappa}{2} \operatorname{Tr}\left(V_{\mu \nu}^{s} \Delta_{C}\right) B^{\mu \nu}, \\
\mathscr{L}_{C}^{\text {mass }} & =\left(m^{2}+\lambda H^{\dagger} H\right) \operatorname{Tr}\left(V^{a ; \mu} V_{\mu}^{a ; \dagger}+\zeta_{1} V_{\mu}^{a} \Delta_{C} V_{\mu}^{a ; \dagger}+\zeta_{2} \Delta_{C} V_{\mu}^{a} \Delta_{C} V_{\mu}^{a ; \dagger}+\cdots\right), \\
\mathscr{L}_{C}^{\text {int }} & =-\beta \operatorname{Tr}\left(V_{\mu}^{s} \Delta_{C}\right) H^{\dagger} D^{\mu} H,
\end{aligned}
$$

where $a=o, s$ stands for the octet and singlet sub-cases and $V_{\mu \nu}^{s}=\partial_{\mu} V_{\nu}^{s}-\partial_{\nu} V_{\mu}^{s}$. The trace is over flavor space. In the color octet case, a covariant derivative is needed and the trace extends to color space. Note that the flavor symmetry ensures that the flavor basis of the vector fields that couple through the operators shown is the same as the flavor basis obtained after rotating to the quark mass field basis in $\mathscr{L}^{\text {Yuk }}$, i.e., that no further flavor violation is present due to a misalignment of the flavor eigenbases.

We have included explicit insertions of Yukawa matrices in $\mathscr{L}_{C}^{\text {mass }}$ as an illustrative example. A series of flavor breaking insertions are also possible on the other terms in the Lagrangian inside the trace. Using the formalism of MFV, the insertions are parameterized by a series in powers of $\Delta_{C}=\left\{Y_{d} Y_{d}^{\dagger}, Y_{u} Y_{u}^{\dagger}, Y_{u}^{\dagger} Y_{u}\right\}$ for $C=\{V, V I, V I I I\}$. For flavor breaking insertions in the kinetic terms, we can always re-diagonalize the fields with a finite renormalization and we neglect the resulting mass splittings, implicitly absorbing these splittings into the leading order mass definition for each flavor. The insertion of Yukawa matrices into the mass terms causes mass splittings among the different flavors. We retain the leading flavor breaking due to the top or bottom Yukawa leading to the mass spectrum

$$
m_{1,2,3}^{2}=m^{2}+\frac{\lambda}{2} v^{2}, \quad m_{4,5,6,7}^{2}=m_{1}^{2}\left(1+\frac{\zeta_{1}}{2} y^{2}\right), \quad m_{8}^{2}=m_{1}^{2}\left(1+\frac{2 \zeta_{1}}{3} y^{2}+\frac{2 \zeta_{2}}{3} y^{4}\right) .
$$

Here we have used the conventions $\langle H\rangle=v / \sqrt{2}$ and $\operatorname{Tr}\left(\tau^{a} \tau^{b}\right)=\operatorname{Tr}\left(t^{a} t^{b}\right)=\delta^{a b} / 2$.

$\mathscr{L}_{C}^{\text {int }}$ arises only in the color singlet case, and is suppressed by an insertion of $\Delta_{C}$. This operator leads to tree level mixing of the SM gauge bosons with the new vector multiplet suppressed by the appropriate Yukawa matrices. When only third generation Yukawa matrices are retained, only the 8 flavor component mixes. For simplicity, we only consider a single mixing with $Z, A$ and $V$, treating $\beta, \kappa \ll 1$ rather than sum the geometric series that results if all insertions of $\Delta_{C}$ are unsuppressed. ${ }^{5}$ We treat this mixing as a perturbation. The fields are transformed to a new field basis $\tilde{V}_{8}, \tilde{B}$ with diagonalized kinetic terms in the presence of the kinetic mixing between the $V^{8}$ colour singlet vector and the $B$ field. The required transformation on the field basis is

$$
\left(\begin{array}{c}
V_{8}^{\alpha} \\
B^{\alpha}
\end{array}\right)=\left(\begin{array}{ccc}
1 & \frac{2 \kappa y^{2}}{\sqrt{3}} \frac{m_{B}^{2}}{m_{B}^{2}-m_{8}^{2}} \\
\frac{2 \kappa y^{2}}{\sqrt{3}} \frac{m_{8}^{2}}{m_{8}^{2}-m_{B}^{2}} & 1
\end{array}\right)\left(\begin{array}{c}
\tilde{V}_{8}^{\alpha} \\
\tilde{B}^{\alpha}
\end{array}\right)
$$

\footnotetext{
${ }^{5}$ One can always reinterpret this parameter to correspond to a series of insertions $\beta \Delta_{C}+\beta^{\prime}\left(\Delta_{C}\right)^{2}+\cdots$.
} 
This transformation leaves the bare tree level mass terms of the $B$ field (after EW symmetry breaking), $m_{B}$, and the tree level mass of the vector field $V_{8}$ unchanged. We neglect the interaction terms in $\mathscr{L}^{\text {Yuk }}$, that are discussed in ref. [11], assuming the direct coupling to the light quarks is small enough that tree level vector exchanges can be neglected, and an oblique EWPD analysis is appropriate. Dijet constraints on the coupling of these fields to light quarks at LHC generically constrain this coupling to be $\sim \mathcal{O}(0.1)$ which is consistent with this assumption. ${ }^{6}$ Consistency of this analysis also requires we neglect the tree level exchanges $\mathcal{O}\left(g^{2} \kappa^{2}\right)$ from the field redefinition of the $B$ field in the covariant derivative in the quark kinetic terms. We also neglect $\mathcal{O}(\beta \kappa)$ contributions in the Lagrangian after the field redefinitions - these effects can be removed by a higher order re-diagonalization of the mass and kinetic operators. With these assumptions, the color singlet vector fields give contributions to the self-energies of the SM gauge bosons,

$$
\Pi_{Z A}\left(p^{2}\right)=0, \quad \Pi_{A A}\left(p^{2}\right)=0, \quad \Pi_{Z Z}\left(p^{2}\right)=\frac{|\beta|^{2} v^{2} m_{Z}^{2} y^{4}}{12\left(p^{2}-m_{8}^{2}\right)} .
$$

Here $y$ is the appropriate Yukawa coupling. Only terms proportional to $g_{\mu \nu}$ are shown.

\subsection{Case VII}

Case VII is a weak singlet, but has non-zero hypercharge, $Y=-1$. We expand the fields in terms of the color Gell-Mann matrices, $\tau^{A} . V_{\mu}^{\dagger} \neq V_{\mu}$ because the $V_{\mu}$ fields are in the $(\overline{3}, 3,1)$ representation of $\mathrm{G}_{\mathrm{F}}$. The fields are flavor bi-fundamentals, $V^{\mu}=\left(V^{\mu}\right)_{j}{ }_{j}$, where $i$ and $j$ are the indices of the $(\overline{3}, 1,1)$ and $(1,3,1)$ representations respectively. The Lagrangians in the mass eigenstate basis are

$$
\begin{aligned}
\mathscr{L}_{V I I} & =\left(1+\delta_{a, o}\right)\left(-\mathscr{L}_{V I I}^{\mathrm{kin}}+\mathscr{L}_{V I I}^{\mathrm{mass}}\right)+\mathscr{L}_{V I I}^{\mathrm{int}}+\mathscr{L}_{V I I}^{\text {c.t. }}+\mathscr{L}_{V I I}^{\mathrm{Yuk}}+\text { h.c. }, \\
\mathscr{L}_{V I I}^{\mathrm{kin}} & =\operatorname{Tr}\left(V^{a ; \mu \nu} V_{\mu \nu}^{a ; \dagger}\right)+i g_{1} \xi B^{\mu \nu} \operatorname{Tr}\left(\left[V_{\mu}^{a}, V_{\nu}^{a ; \dagger}\right]\right), \\
\mathscr{L}_{V I I}^{\mathrm{mass}} & =\left(m^{2}+\lambda H^{\dagger} H\right) \operatorname{Tr}\left(V^{a ; \mu} V_{\mu}^{a ; \dagger}+\zeta_{1} V^{a ; \mu} Y_{u} Y_{u}^{\dagger} V_{\mu}^{a ; \dagger}+\zeta_{2} V^{a ; \mu} Y_{u} Y_{u}^{\dagger} V_{\mu}^{a ; \dagger} Y_{d} Y_{d}^{\dagger}+\cdots\right), \\
\mathscr{L}_{V I I}^{\mathrm{int}} & =-\beta \operatorname{Tr}\left[\left(V_{\mu}^{s} \Delta_{V I I}\right)^{\dagger}\right]\left(D^{\mu} H\right)^{\dagger} \tilde{H},
\end{aligned}
$$

where $V_{\mu \nu}^{s}=D_{\mu} V_{\nu}^{s}-D_{\nu} V_{\mu}^{s}, D_{\mu}=\partial_{\mu}-i g_{1} Y B_{\mu}, \Delta_{V I I}=Y_{u} Y_{d}^{\dagger}$, and $\tilde{H}=i \sigma_{2} H^{\star}$ (and as before a color gauge field term is included in the covariant derivative in the case of color octet vector). The mass splittings for case VII are as follows

$$
\begin{gathered}
m_{11,21,12,22}^{2}=m^{2}+\frac{\lambda}{2} v^{2}, \quad m_{13,31,23,32}^{2}=m_{11}^{2}\left(1+\frac{\zeta_{1}}{2} y_{t}^{2}\right), \\
m_{33}^{2}=m_{11}^{2}\left(1+\frac{\zeta_{1}}{2} y_{t}^{2}+\frac{\zeta_{2}}{2} y_{b}^{2} y_{t}^{2}\right) .
\end{gathered}
$$

$\mathscr{L}^{\text {c.t. }}$ contains all the terms needed to make this theory finite, which includes higherdimensional operators as the theory is non-renormalizable. See section 2.5 for details on

\footnotetext{
${ }^{6}$ However, the coupling of these fields that involve the top quark could be far larger due to flavor splitting effects, allowing these fields to still explain the $A_{\mathrm{FB}}^{t \bar{t}}$ anomaly while this EWPD analysis is appropriate; see [11] for a more detailed discussion.
} 
these operators. The vector field multiplet contributions to the self-energies of the SM gauge bosons are,

$$
\begin{aligned}
& \Pi_{Z Z}\left(p^{2}\right)=D\left(R_{C}\right) s_{w}^{2} g_{1}^{2} \sum_{f=1}^{9}\left[f+\xi g+\xi^{2} h\right]\left(p^{2}, m_{f}^{2}\right), \\
& \Pi_{Z A}\left(p^{2}\right)=-D\left(R_{C}\right) s_{w} c_{w} g_{1}^{2} \sum_{f=1}^{9}\left[f+\xi g+\xi^{2} h\right]\left(p^{2}, m_{f}^{2}\right), \\
& \Pi_{A A}\left(p^{2}\right)=D\left(R_{C}\right) c_{w}^{2} g_{1}^{2} \sum_{f=1}^{9}\left[f+\xi g+\xi^{2} h\right]\left(p^{2}, m_{f}^{2}\right), \\
& \Pi_{W W}\left(p^{2}\right)=\delta_{a, s} \frac{y_{t}^{2} y_{b}^{2}|\beta|^{2} v^{2} m_{W}^{2}}{8\left(p^{2}-m_{33}^{2}\right)}
\end{aligned}
$$

where $D\left(R_{C}\right)$ is the dimension of the color representation. Here $s_{w}, c_{w}$ are the sine and cosine of the weak mixing angle with convention $e=g_{1} / c_{w}$, and $g_{1}$ is the hypercharge coupling. The form factors $f, g, h$ are defined in the appendix A.

\section{$2.3 \quad$ Case IX}

Case IX is a weak triplet that has zero hypercharge. In addition to being parameterized by $\tau^{A}$ and $t^{B}$ for color and flavor, the fields are also parameterized using the Pauli matrices, $V_{\mu}=\sigma_{i} V_{\mu}^{i}$, for the $\mathrm{SU}_{\mathrm{L}}(2)$ representation. We suppress the color singlet, octet label on the field in this section for clarity ${ }^{7}$ while including the $\mathrm{SU}_{\mathrm{L}}(2)$ index, the Lagrangians are

$$
\begin{aligned}
\mathscr{L}_{I X} & =\left(1+\delta_{a, o}\right)\left(-\mathscr{L}_{I X}^{\mathrm{kin}}+\mathscr{L}_{I X}^{\mathrm{mass}}\right)+\mathscr{L}_{I X}^{\mathrm{int}}+\mathscr{L}_{I X}^{\mathrm{ct.t}}+\mathscr{L}_{I X}^{\mathrm{yuk}}+\text { h.c. } \\
\mathscr{L}_{I X}^{\mathrm{kin}} & =\frac{1}{2} \operatorname{Tr}\left(V_{i}^{\mu \nu} V_{\mu \nu}^{i ; \dagger}\right)+\frac{\kappa}{2} \operatorname{Tr}\left(V_{\mu \nu}^{i} \Delta_{I X}\right) W_{i}^{\mu \nu}-g_{2} \xi \epsilon_{i j k} W^{i ; \mu \nu} \operatorname{Tr}\left(V_{\mu}^{j} V_{\nu}^{k \dagger}\right), \\
\mathscr{L}_{I X}^{\mathrm{mass}} & =\left(m^{2}+\lambda H^{\dagger} H\right) \operatorname{Tr}\left(V_{i}^{\mu} V_{\mu}^{i ; \dagger}+\zeta_{1} V_{i}^{\mu} \Delta_{I X} V_{\mu}^{i \dagger}+\zeta_{2} \Delta_{I X} V_{i}^{\mu} \Delta_{I X} V_{\mu}^{i ; \dagger}+\cdots\right), \\
\mathscr{L}_{I X}^{\text {int }} & =-\beta \operatorname{Tr}\left(V_{\mu}^{i} \Delta_{I X}\right) H^{\dagger} \sigma_{i} D^{\mu} H
\end{aligned}
$$

where $V_{\mu \nu}^{i}=\left(D_{\mu} V_{\nu}\right)^{i}-\left(D_{\nu} V_{\mu}\right)^{i}, D_{\mu}^{i j}=\partial_{\mu} \delta^{i j}-g_{2} \epsilon^{i j k} W_{k \mu}$, and $\Delta_{I X}=Y_{u}^{\dagger} Y_{u}$. The mass splittings are the same as in Case VIII. In this section we neglect the effects of the kinetic mixing operator considering the case $\kappa \ll \beta, g_{2} \xi, g_{2} \cdot{ }^{8}$

\footnotetext{
${ }^{7}$ The $\kappa, \beta$ operators are only for the singlet case as before.

${ }^{8}$ The effect of the kinetic mixing with a nonabelian field has recently been studied in $[25,26]$ for example and our results can be directly extended to include kinetic mixing. Many of the effects of kinetic mixing can be absorbed into a redefinition of the remaining unknown parameters of this model once a diagonalization of the kinetic terms is undertaken. The transformation to canonical kinetic terms is exactly of the form given in section 2.1 for each isospin state when the kinetic mixing is not neglected.
} 
The contributions to the self-energies of the SM gauge bosons are then

$$
\begin{aligned}
\Pi_{Z Z}\left(p^{2}\right) & =2 D\left(R_{C}\right) c_{w}^{2} g_{2}^{2} \sum_{f=1}^{8}\left[f+\xi g+\xi^{2} h\right]\left(p^{2}, m_{f}^{2}\right)+\delta_{a, s} \frac{|\beta|^{2} y_{t}^{4} v^{2} m_{Z}^{2}}{12\left(p^{2}-m_{8}^{2}\right)}, \\
\Pi_{Z A}\left(p^{2}\right) & =2 D\left(R_{C}\right) s_{w} c_{w} g_{2}^{2} \sum_{f=1}^{8}\left[f+\xi g+\xi^{2} h\right]\left(p^{2}, m_{f}^{2}\right) \\
\Pi_{A A}\left(p^{2}\right) & =2 D\left(R_{C}\right) s_{w}^{2} g_{2}^{2} \sum_{f=1}^{8}\left[f+\xi g+\xi^{2} h\right]\left(p^{2}, m_{f}^{2}\right) \\
\Pi_{W W}\left(p^{2}\right) & =2 D\left(R_{C}\right) g_{2}^{2} \sum_{f=1}^{8}\left[f+\xi g+\xi^{2} h\right]\left(p^{2}, m_{f}^{2}\right)+\delta_{a, s} \frac{|\beta|^{2} y_{t}^{4} v^{2} m_{W}^{2}}{3\left(p^{2}-m_{8}^{2}\right)}
\end{aligned}
$$

\subsection{Cases X and XI}

These fields are $\mathrm{SU}_{\mathrm{L}}(2)$ doublets, flavor bi-fundamentals, and are in either the color sextet or anti-triplet representations, $a=6, \overline{3}$. The only difference between the two cases is they have different hypercharges, $Y_{C}=\{-1 / 6,5 / 6\}$ for $C=\{X, X I\}$. The Lagrangians are

$$
\begin{aligned}
\mathscr{L}_{C}= & -\mathscr{L}_{C}^{\text {kin }}+\mathscr{L}_{C}^{\text {mass }}+\mathscr{L}_{C}^{\text {c.t. }}+\mathscr{L}_{C}^{\text {yuk }}+\text { h.c., } \\
\mathscr{L}_{C}^{\text {kin }}= & \frac{1}{2} V^{a ; \mu \nu} V_{\mu \nu}^{a ; \dagger}+i g_{1} \xi_{1} B^{\mu \nu} V_{\mu}^{a} V_{\nu}^{a ; \dagger}+i g_{2} \xi_{2} V_{\mu}^{a} W^{\mu \nu} V_{\nu}^{a ; \dagger}, \\
\mathscr{L}_{C}^{\text {mass }}= & \left(m^{2}+\lambda_{1} H^{\dagger} H\right)\left(V^{a ; \mu} V_{\mu}^{a ; \dagger}+\zeta_{1} V^{a ; \mu} \Delta_{C} V_{\mu}^{a ; \dagger}+\cdots\right) \\
& \quad+\lambda_{2}\left(H^{\alpha \dagger} V_{\alpha}^{a ; \mu} V_{\mu}^{a ; \beta \dagger} H_{\beta}+\cdots\right)+\lambda_{3}\left(\tilde{H}^{\alpha \dagger} V_{\alpha}^{a ; \mu} V_{\mu}^{a ; \beta \dagger} \tilde{H}_{\beta}+\cdots\right),
\end{aligned}
$$

where $V_{\mu \nu}^{6}=D_{\mu} V_{\nu}^{6}-D_{\nu} V_{\mu}^{6}, D_{\mu}=\partial_{\mu}-i g_{3} \tau_{6}^{A} A_{\mu}^{A}-i g_{2} \sigma^{i} W_{\mu}^{i}-i g_{1} Y B_{\mu}$, and $\Delta_{C}=Y_{u} Y_{u}^{\dagger}$ in both cases. In the last line of $\mathscr{L}_{C}^{\text {mass }}$ the weak indices, $\alpha$ and $\beta$, are explicit. We did not explicitly write down in the Lagrangian the flavor breaking insertions in the additional Higgs terms that appear in the mass splittings below. In these cases there is a mass splitting in the electroweak doublet

$$
m_{Q+}^{2}=m^{2}+\left(\lambda_{1}+\lambda_{2}\right) \frac{v^{2}}{2}, m_{Q-}^{2}=m^{2}+\left(\lambda_{1}+\lambda_{3}\right) \frac{v^{2}}{2}
$$

where $Q \pm=Y \pm 1 / 2$. Note that if there were no mass splitting between the weak states then there would to no contribution to $\Pi_{W^{3} B}$ because the interaction has the form $\operatorname{Tr}\left(V_{\mu}^{\dagger} \sigma^{3} V^{\mu}\right)$. In addition there is the usual mass splitting in flavor space

$$
m_{11,12,21,22}^{2}=m_{Q \pm}^{2}, m_{13,23,32,31,33}^{2}=m_{Q \pm}^{2}\left(1+\zeta y_{t}^{2}\right)
$$


The contribution of the vector fields to the self-energies of the SM gauge bosons are written in the electroweak basis in this case to reduce clutter,

$$
\begin{aligned}
\Pi_{W^{3} W^{3}}\left(p^{2}\right) & =D\left(R_{C}\right) \frac{g_{2}^{2}}{4} \sum_{f, L}\left[f+\xi_{2} g+\xi_{2}^{2} h\right]\left(p^{2}, m_{f, L}^{2}\right), \\
\Pi_{W^{3} B}\left(p^{2}\right) & =D\left(R_{C}\right) \frac{g_{2} g_{1} Y}{2} \sum_{f, L}(-1)^{L-1}\left[f+\frac{1}{2}\left(\xi_{1}+\xi_{2}\right) g+\xi_{1} \xi_{2} h\right]\left(p^{2}, m_{f, L}^{2}\right), \\
\Pi_{B B}\left(p^{2}\right) & =D\left(R_{C}\right) g_{1}^{2} Y^{2} \sum_{f, L}\left[f+\xi_{1} g+\xi_{1}^{2} h\right]\left(p^{2}, m_{f, L}^{2}\right), \\
\Pi_{W^{1} W^{1}}\left(p^{2}\right) & =D\left(R_{C}\right) \frac{g_{2}^{2}}{2} \sum_{f}\left[f+\xi_{2} g+\xi_{2}^{2} h\right]\left(p^{2}, m_{f, Q+}^{2}, m_{f, Q-}^{2}\right),
\end{aligned}
$$

where the sums over flavor $f$ and weak $L$ states run from 1 to 9 and 2 respectively. For contributions from $\Pi_{W^{3} B}$, it is the difference of weak states rather than the sum that contributes. The factor of $(-1)^{L-1}$ accounts for this. For $\Pi_{W W}$, there is no sum over weak state because both particles of the weak doublet need to be in the loop to conserve electric charge. Note that the mass splitting among weak states causes $\Pi_{W W}$ to be a function of both masses.

The $S$ parameter is negative in certain regions of parameter space for cases X and XI. For example, with $\xi_{1}=\xi_{2}=0$ and $m_{ \pm}^{2} \gg m_{Z}^{2}, S \propto Y_{C} \ln \left(m_{+}^{2} / m_{-}^{2}\right)$.

\subsection{Counterterms and higher-dimensional operators}

The vectors are effective fields, and the Lagrangians contain an infinite number of nonrenomalizable operators. At low scales, $p$, compared to the cutoff scale of the effective theory, $\Lambda$, the contribution of these terms to self-energies is suppressed by powers of $(p / \Lambda)^{n}$. The ratio of scales can be set by the external momentum $p^{2} / \Lambda^{2}$ or can be set by the ratio of other invariants $m_{v}^{2} / \Lambda^{2}$ depending on the operator of interest. Because of this suppression we neglect contributions to the self-energies from almost all of these other operators. The reason why we did not neglect the contributions from all of the higher dimensional operators is explained in what follows.

Being psuedo-obersevables, the STUVWX parameters must be free of divergences and independent of the renormalization point $\mu$. Contributions to the self-energies have two origins. Contributions of the first kind contain no powers of momentum in the numerator from internal propagators. As expected, the resulting STUVWX parameters are finite and $\mu$-independent. These are the only terms that would be found in a renormalizable theory. There is no need to add a field renormalization term, such as $Z_{B} B^{\mu \nu} B_{\mu \nu}$, to the Lagrangian because its contribution to each of these parameters is identically zero.

The second type of contributions contain all of the other terms that come about from at least one propagator's $p_{\mu} p_{\nu}$ piece. In this case, contributions to the STUVWX parameters are divergent and require higher-dimensional operators as counter terms. $\mathscr{L}_{C}^{\text {c.t. }}$ contains all the counter terms necessary to absorb the divergences of the self-energies from the dimension- 4 operators. Not all of the operators are needed as counterterms in each case. 


\begin{tabular}{|c|c|}
\hline Oblique & $y \pm \sigma$ \\
\hline $\mathrm{S}$ & $0.07 \pm 0.41$ \\
$\mathrm{~T}$ & $-0.40 \pm 0.28$ \\
$\mathrm{U}$ & $0.65 \pm 0.33$ \\
$\mathrm{~V}$ & $0.43 \pm 0.29$ \\
$\mathrm{~W}$ & $3.0 \pm 2.5$ \\
$\mathrm{X}$ & $-0.17 \pm 0.15$ \\
\hline
\end{tabular}

Table 2. EWPD fit to STUVWX results of ref. [23]. $y$ is the best-fit value, and $\sigma$ is the square root of the diagonal element of the determined covariance matrix.

With this understanding, the counterterms are

$$
\begin{aligned}
\mathscr{L}_{C}^{\text {c.t. }}= & \frac{Z_{1}}{\Lambda^{2}} \partial_{\rho} B_{\mu \nu} \partial^{\rho} B^{\mu \nu}+\frac{Z_{2}}{\Lambda^{4}} \partial_{\tau} \partial_{\rho} B_{\mu \nu} \partial^{\tau} \partial^{\rho} B^{\mu \nu}+\frac{Z_{3}}{\Lambda^{2}}\left|H^{\dagger} D_{\mu} H\right|^{2} \\
& +\frac{Z_{4}}{\Lambda^{2}} \operatorname{Tr}\left(D_{\rho} W_{\mu \nu} D^{\rho} W^{\mu \nu}\right)+\frac{Z_{5}}{\Lambda^{4}} \operatorname{Tr}\left(D_{\tau} D_{\rho} W_{\mu \nu} D^{\tau} D^{\rho} W^{\mu \nu}\right) \\
& +\frac{Z_{6}}{\Lambda^{2}} H^{\dagger} W_{\mu \nu} H B^{\mu \nu}+\frac{Z_{7}}{\Lambda^{4}} H^{\dagger} D_{\rho} W_{\mu \nu} H \partial^{\rho} B^{\mu \nu}+\frac{Z_{8}}{\Lambda^{6}} H^{\dagger} D_{\tau} D_{\rho} W_{\mu \nu} H \partial^{\tau} \partial^{\rho} B^{\mu \nu}
\end{aligned}
$$

The divergences come in the form $2 / \varepsilon-\gamma+\ln \left(4 \pi \mu^{2} / m^{2}\right)$ (using dimensional-regularization in $D=4-\varepsilon$ dimensions) and we use the operators to cancel the divergences and $\mu$ dependence. In practice, we take $\mu=1 \mathrm{TeV}$ for numerical evaluations and include in our fit a finite contribution from one higher-dimensional operator to absorb the $\mu$-dependence when a divergence is canceled by a higher dimensional operator.

\section{$3 \quad$ Fit to electroweak precision data}

Electroweak precision data provides strong constraints on the MFV vectors under consideration. A convenient subset of the full set of corrections are the oblique corrections. Purely NP contributions to the self-energies of the electroweak gauge bosons can be written in the following form $\Pi_{a b}^{\mu \nu}(k)=\Pi\left(k^{2}\right)_{a b} g^{\mu \nu}+f\left(k^{2}\right) k^{\mu} k^{\nu}$ where $a b=\left\{W^{+} W^{-}, Z Z, A A, Z A\right\}$. When the masses of the new states are heavy compared to the EWSB scale, the vacuum polarizations can be expanded in momentum $\Pi_{a b}\left(q^{2}\right) \approx \Pi_{a b}(0)+q^{2} \Pi_{a b}^{\prime}(0)$ and the STU parameters can be used. However, when one wishes to consider masses comparable to (or smaller than) the EWSB scale, this expansion in momentum becomes invalid. In that case, the STUWVX parameters (defined in [20]), which do not expand in $v^{2} / m_{\mathrm{NP}}^{2}$, can be used and can be more constraining. The theoretical predictions of EWPD of the 2008 PDG [24] was used to perform a modern fit to the STUVWX parameters in ref. [23]. The results of the fit are given in table 2. The correlation coefficient matrix of the fit is given by

$$
\rho=\left(\begin{array}{cccccc}
1 & 0.60 & 0.38 & -0.57 & 0 & -0.86 \\
0.60 & 1 & -0.49 & -0.95 & 0 & -0.13 \\
0.38 & -0.49 & 1 & 0.46 & -0.01 & -0.76 \\
-0.57 & -0.95 & 0.46 & 1 & 0 & 0.13 \\
0 & 0 & -0.01 & 0 & 1 & 0 \\
-0.86 & -0.13 & -0.76 & 0.13 & 0 & 1
\end{array}\right) .
$$



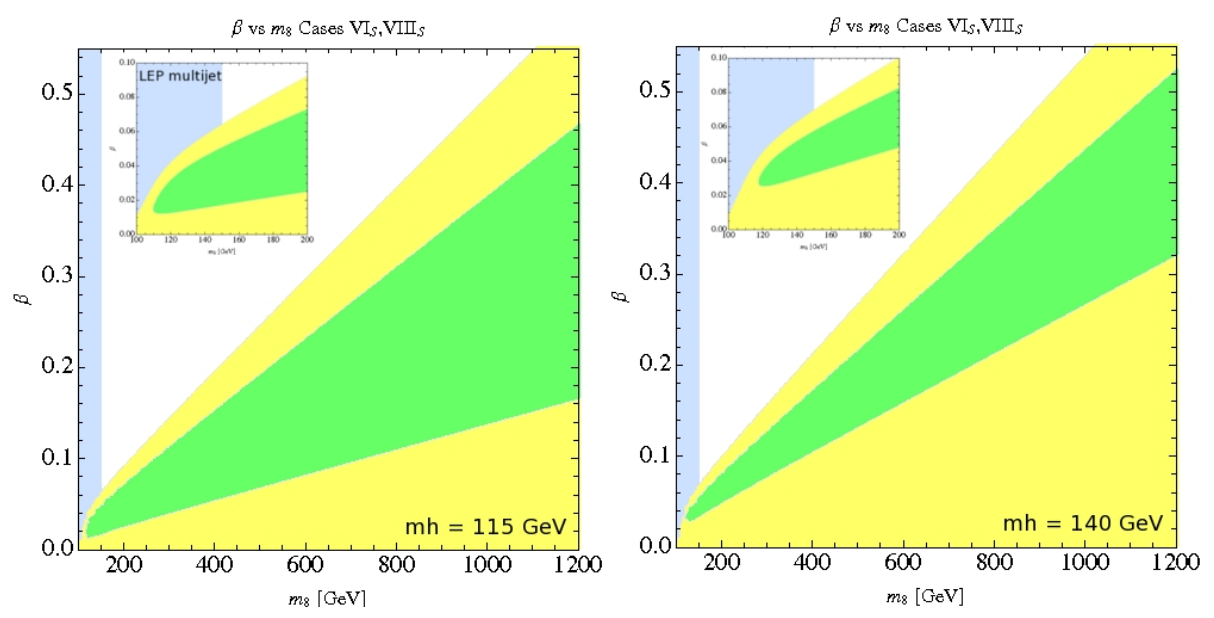

Figure 1. In all of the plots, the green and yellow regions represent regions of parameter space that are allowed at 1- and 2- $\sigma$ respectively. Also shown is the approximate bound from LEP due to multijet final states when the coupling to light quarks is not neglected. Note that these bounds are complementary in that the oblique analysis fails in this case.

The least-squared estimators $\hat{\boldsymbol{\theta}}$ for the set of parameters $\boldsymbol{\theta}$ of a given model, is determined by the minimum of

$$
\chi^{2}(\boldsymbol{\theta})=(\mathbf{y}-\mathbf{F}(\boldsymbol{\theta}))^{T} V^{-1}(\mathbf{y}-\mathbf{F}(\boldsymbol{\theta}))
$$

where $\mathbf{y}$ is a vector of the best-fit values of the STUVWX parameters, $V^{-1}$ is the inverse of the covariance matrix $V_{i j}=\sigma_{i} \rho_{i j} \sigma_{j}$, and $\mathbf{F}(\boldsymbol{\theta})$ is the corresponding vector of predicted values of the model. We determine the 1- and 2- $\sigma$ confidence regions of allowed parameter space by requiring that the contribution of NP to $\chi^{2}(\boldsymbol{\theta})$ satisfy

$$
\chi^{2}(\boldsymbol{\theta}) \leq \chi_{\min }^{2}+\Delta \chi^{2}
$$

where $\Delta \chi^{2}$ corresponds to the probability that the confidence region of parameter space determined with the cumulative distribution function and contains the best fit value of the parameters.

\section{Results}

In this section, constraints on flavor symmetric vectors are discussed on a case-by-case basis. Mass splittings among flavor states are generally assumed to be small and not relevant for these constraints. This follows from the fact that there are no loops with different flavor multiplet species to the approximation we work to, so the breaking of $\mathrm{G}_{\mathrm{F}}$ is not linked to the breaking of custodial symmetry $\mathrm{SU}_{\mathrm{C}}(2)$. Note however that in certain cases, such as the constraint plots for section A, what is plotted is the constraint space for $\beta$ and $m_{8}$. The other vectors in these multiplets are split in general from the mass $m_{8}$ with a splitting of $\mathcal{O}\left(\zeta_{1,2}\right)$ and the appropriate Yukawa suppression. 

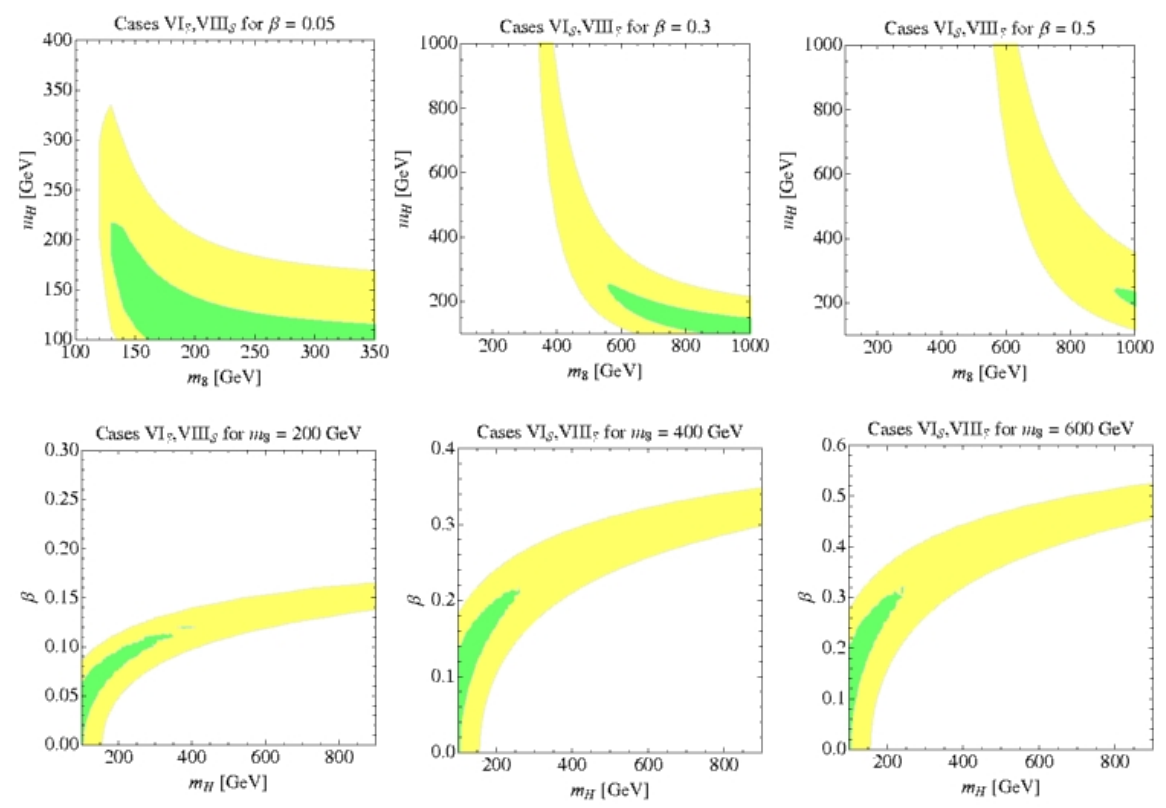

Figure 2. Allowed confidence regions at 1- and 2- $\sigma$ when the Higgs mass is raised through a $\mathrm{SU}_{\mathrm{C}}(2)$ violating operator - due to coupling to a flavor symmetric vector multiplet.

\subsection{Cases V, VI, and VIII}

In all of these color octet cases, EWPD does not place limits on the parameters of the model in the approximation that we are working to - the relevant operator is forbidden. However, the absence of vector pair-production at LEP implies a kinematic bound for color singlet and octet vectors of at least $m_{V} \gtrsim 105 \mathrm{GeV}$. When the direct coupling of the vectors to quarks is $\mathcal{O}(1)$, the experimental bound become stronger, $m_{V} \gtrsim 150 \mathrm{GeV}$ from anomalous multi-jet events at LEP [11]. We also include the latter bound in the figure. Dedicated collider searches can significantly raise these mass bounds.

The bounds from EWPD on case $V_{\mathrm{s}}$ are particularly weak as $y_{b} \ll 1$ and the other parameters in this model are unconstrained. In theories such as the large $\tan \beta$ limit of the MSSM where $y_{b}$ becomes $\mathcal{O}(1)$, the constraints are similar to cases $\mathrm{VI}_{\mathrm{s}}$ and $\mathrm{VIII}_{\mathrm{s}}$. In figure 1 the Higgs mass is fixed to $m_{h}=115(140) \mathrm{GeV}$ in the left (right) figure with its one loop contribution to the EW parameters floated from a reference value of $\hat{m}_{h}=96 \mathrm{GeV}$ in the fit.

Operators such as $\operatorname{Tr}\left(V_{\mu}^{s} \Delta_{C}\right) H^{\dagger} D^{\mu} H$ lead to a violation of $\mathrm{SU}_{\mathrm{C}}(2)$ and can act to raise the Higgs mass by giving a positive contribution to the $T$ parameter. We illustrate the effect on the best fit value of the Higgs mass in figure 2. The entire light mass region of the Higgs in the SM may be excluded in the near future by CMS and/or ATLAS or a combination of the experimental data sets. Simple (flavor safe) mechanisms to raise the Higgs mass in the EWPD as demonstrated here would then be of greater interest. This mechanism also exists in several of the remaining cases when an operator of this form is allowed. We will generally fix the Higgs mass in what follows to reduce the parameter space. 

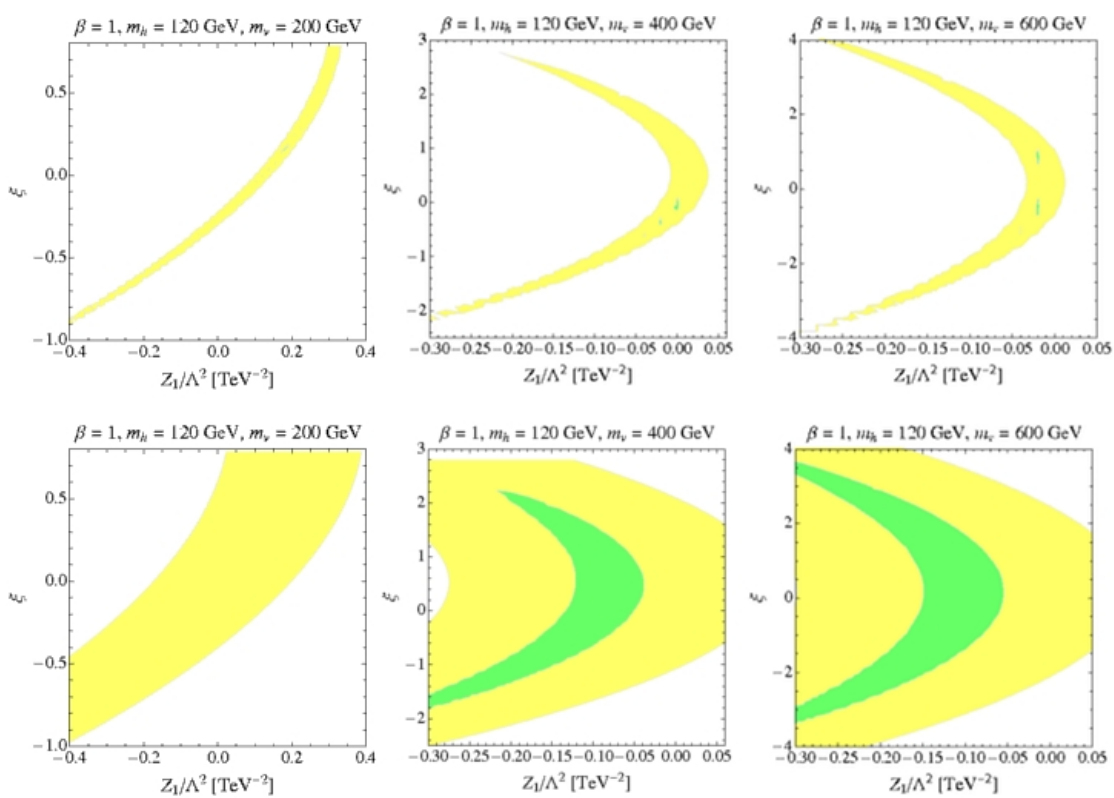

Figure 3. Space of allowed $\xi$ and $Z_{1} / \Lambda^{2}$ for cases $\mathrm{VII}_{\mathrm{o}}$ (top) and $\mathrm{VII}_{\mathrm{s}}$ (bottom). Here we have set $\zeta_{1,2}=0$, neglecting flavour breaking. The regions shown are weakly dependent on variations in $\beta$, we have fixed $\beta=1$ and $m_{h}=120 \mathrm{GeV}$. Left to right the masses are $m_{v}=200,400,600 \mathrm{GeV}$.

\subsection{Case VII}

Eliminating the $\mu$-dependence from STUVWX determines a relationship between $\xi$ and $Z_{1} / \Lambda^{2}$ as a function of the other (allowed) parameters in the model. For numerical purposes, we ignore the dimension- 8 operator with coefficient $Z_{2}$. Figure 3 shows the relationships between $\xi$ and $Z_{1} / \Lambda^{2}$ for various masses.

The allowed regions in the multidimensional parameter space has a nontrivial dependence on the various parameters, as is further illustrated in figure 4. Generically the parameter space for $\mathrm{VII}_{\mathrm{S}}$ is less constrained than $\mathrm{VII}_{\mathrm{O}}$. Both cases require a strong correlation between the finite part of the counter term and the remaining parameters to not be ruled out.

\subsection{Case IX}

The operator proportional to $\beta$ again leads to a relaxation of the Higgs mass bounds as in Cases $\mathrm{VI}_{\mathrm{s}}$, VIII . We set $\beta=0$ and $m_{h}=115 \mathrm{GeV}$ in what follows and examine the remaining parameter space. This case has the strongest constraints from oblique EWPD. A strong correlation is required between $\xi, m$ and $Z_{4} / \Lambda^{2}$ for the allowed parameter space. This is illustrated in figure 5 for IX $_{\mathrm{s}}$. We do not find viable parameter space for $\operatorname{IX}_{\mathrm{o}}$.

\subsection{Cases X and XI}

There is no operator proportional to $H^{\dagger} D^{\mu} H$ in these cases that directly violates $\mathrm{SU}_{\mathrm{C}}(2)$ and allows the Higgs mass to be raised. In cases $\mathrm{X}_{6, \overline{3}}$ the allowed parameter space dependence on the parameter $\xi_{1}$ is trivial, not showing a significant correlation with $Z / \Lambda^{2}, \xi_{2}$ 

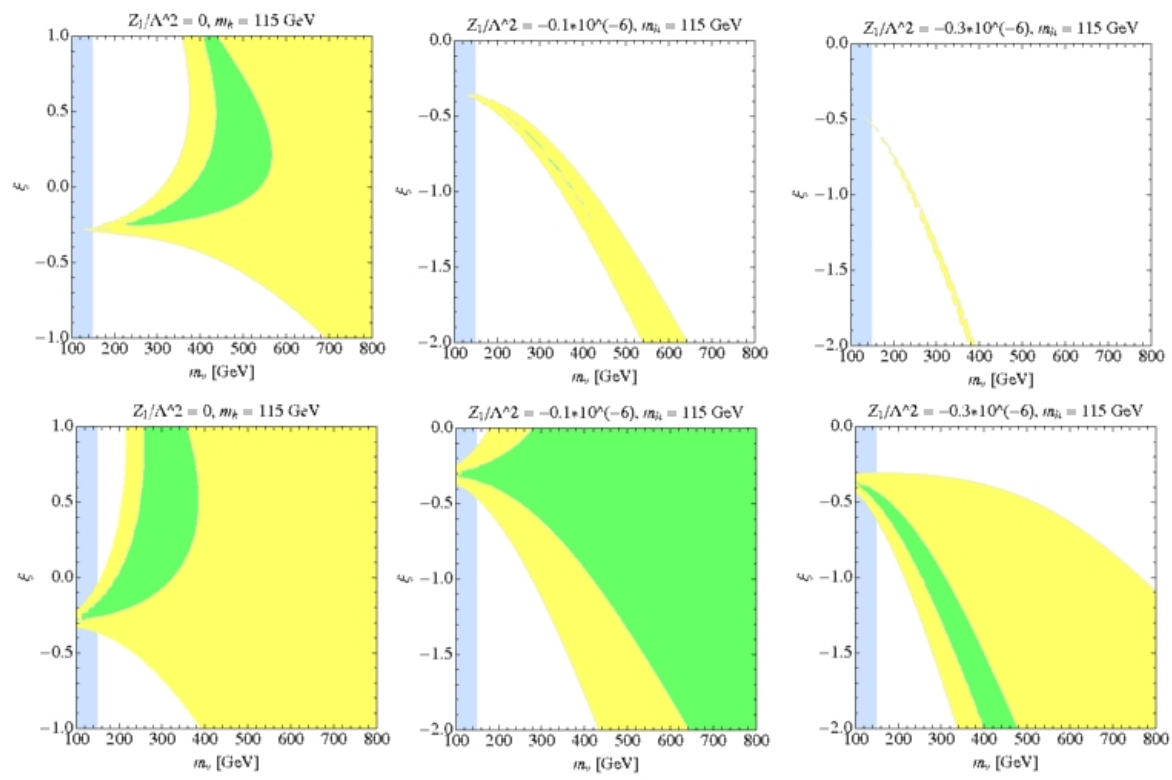

Figure 4. Space of allowed $\xi$ and $m_{V}$ for cases $\mathrm{VII}_{\mathrm{o}}$ (top) and $\mathrm{VII}_{\mathrm{s}}$ (bottom). Here we neglecting flavor breaking and $\beta$ as the allowed parameter space is weakly dependent on these parameters. We have set $m_{h}=115 \mathrm{GeV}$. Left to right the counterterm values are $Z_{1} / \Lambda^{2}=(0,-0.1,-0.3) \times \mathrm{TeV}^{-2}$.
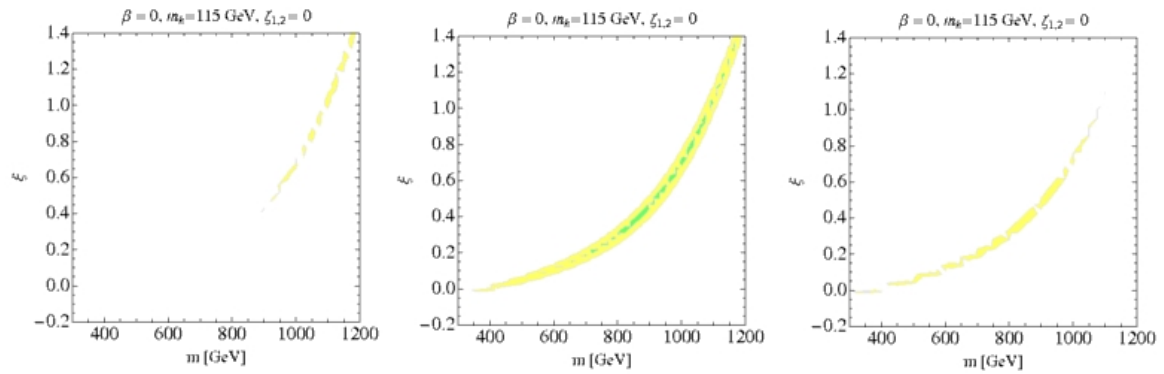

Figure 5. Space of allowed $\xi$ and $m$ for case $\mathrm{IX}_{\mathrm{s}}$. Here we have set $\beta=0$ and used $m_{h}=115 \mathrm{GeV}$. Left to right the counterterm is $Z_{4} / \Lambda^{2}=(-3,0,3) \times 10^{-2} \mathrm{TeV}^{-2}$.

or $m_{V}$. The correlation between $\xi_{2}$ and $Z / \Lambda^{2}$ in the allowed parameter space is shown in figure 6 . The allowed masses in a joint fit with fixed $m_{h}=115 \mathrm{GeV}$ is shown in figure 7 for model $\mathrm{X}_{\overline{3}}$. For comparison the required correlations between the parameters for case $\mathrm{XI}_{\overline{3}}$ are shown in figure 8.

\section{Conclusions}

In this paper we have examined the constraints that oblique EWPD places on flavor symmetric vector fields. We have examined these constraints on the vector field multiplets that 

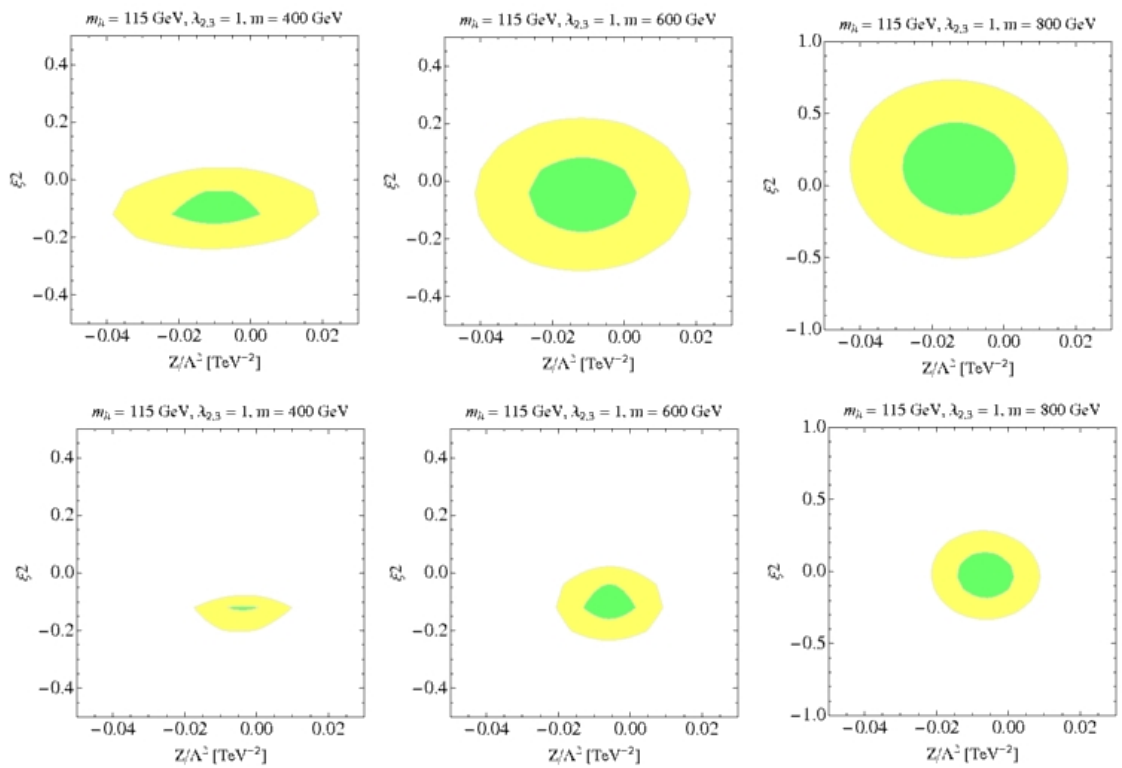

Figure 6. Space of allowed $\xi_{2}$ and $Z / \Lambda^{2}$ for cases $\mathrm{X}_{\overline{3}}$ (top) and $\mathrm{X}_{6}$ (bottom). Here we neglect flavour breaking and $\xi_{1}$. Breaking of the mass degeneracy of the $\mathrm{SU}_{\mathrm{L}}(2)$ states is included, again the effect on the parameter space is negligible. Left to right the masses are $m_{v}=400,600,800 \mathrm{GeV}$.

transform under the flavor group and couple to quark bi-linears at tree level, while not initially breaking the quark global flavor symmetry group. These extensions to the SM are treated as effective fields in fitting to oblique EWPD, including appropriate counterterms to make the EWPD pseudo-observables finite and renormalization scale independent when required. We have found that large regions of parameter space exist where a joint fit to these fields with the Higgs allows a good fit, while the masses of the vector multiplets are $\sim v$. Vectors of this form can act to significantly relax the mass bound on the Higgs in a flavor safe manner, as we have demonstrated in detail for Cases $\mathrm{VI}_{\mathrm{s}}$, and $\mathrm{VIII}_{\mathrm{s}}$. Flavor safe mechanisms to raise the Higgs mass bound may be of greater interest if the entire light Higgs mass parameter space is excluded experimentally in the near future.

Conversely, it is interesting to note that large regions of parameter space exist in the models where the Higgs mass is in the light mass region with $m_{h} \sim 115 \mathrm{GeV}$, joint fits to EWPD are improved over the SM alone, and field content is allowed that could possibly explain the $A_{\mathrm{FB}}^{t \bar{t}}$ anomaly. Vector fields of the form we have considered are relatively unconstrained by indirect searches in flavor physics due to their flavor symmetry, and have been shown to be consistent with oblique EWPD constraints. Further dedicated studies of the constraints on these flavour multiplets from non-oblique precision EW observables, such as $R_{b}$, may provide stronger constraints on the allowed mass scales. Dedicated direct collider studies of flavor safe vector fields also have to potential to raise the mass bounds on these models, or discover models of this form at LHC. 

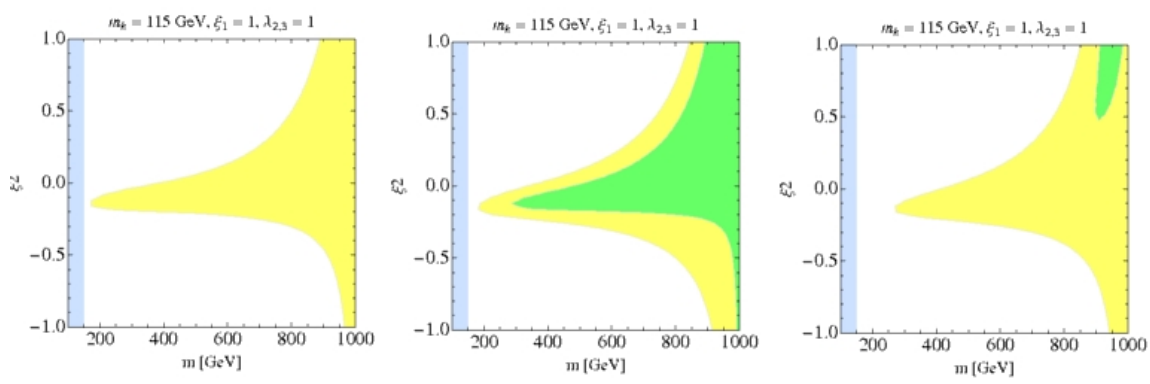

Figure 7. Space of allowed $\xi_{2}$ and $m$ for $\mathrm{X}_{\overline{3}}$. Flavour breaking is neglected and we set $\xi_{1}=1$ and $\lambda_{2,3}=1$ - the dependence on these parameters is negligible. We have also set $m_{h}=115 \mathrm{GeV}$. Left to right $Z / \Lambda^{2}=(0.01,-0.01,-0.03) \mathrm{TeV}^{-2}$. For case $\mathrm{X}_{6}$ the parameter space with $Z / \Lambda^{2}=$ $-0.01 \mathrm{TeV}^{-2}$ is similar, while there is no allowed parameter space for the other values of $Z / \Lambda^{2}$.
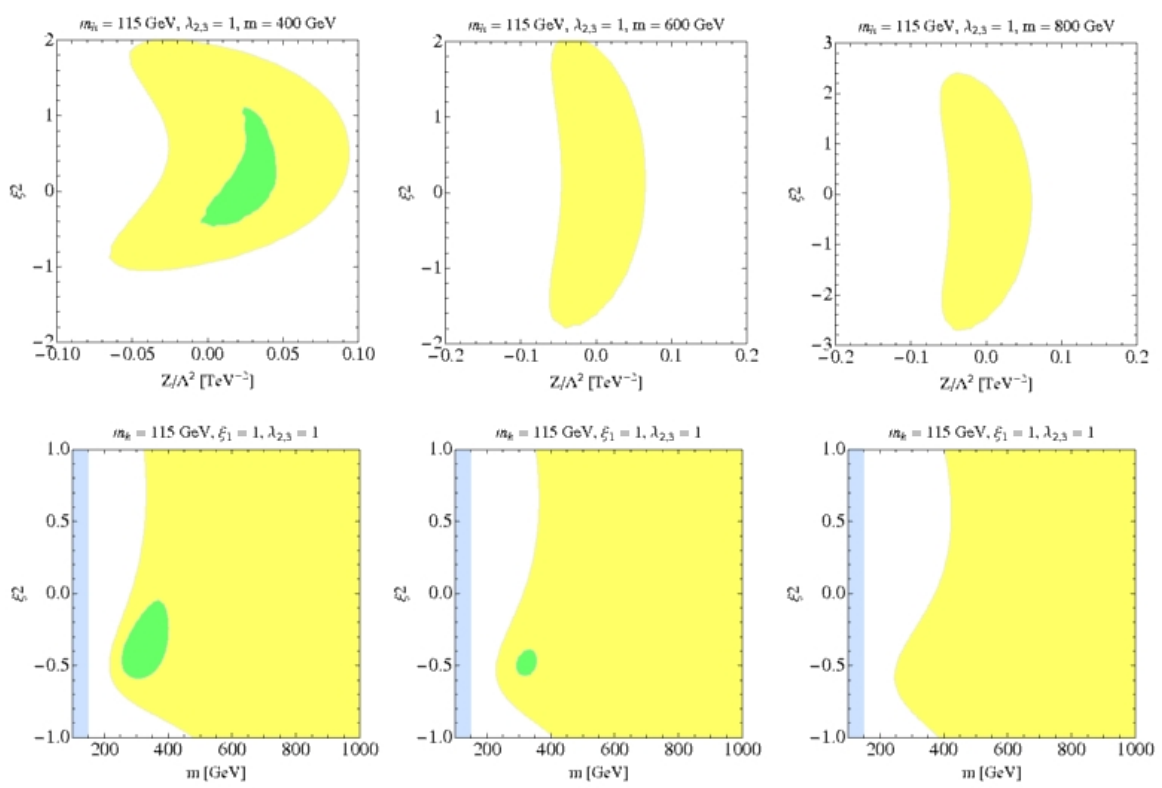

Figure 8. Space of allowed $\xi_{2}$ and $Z / \Lambda^{2}$ for case $\mathrm{XI}_{\overline{3}}$ (top), left to right the masses are $m_{v}=$ $400,600,800 \mathrm{GeV}$, other parameters same as above, $Z / \Lambda^{2}$ same as above left to right for $\mathrm{XI}_{\overline{3}}$ (bottom row).

\section{Acknowledgments}

The work of B.G. and C.M. was supported in part by the US Department of Energy under contract DOE-FG03-97ER40546. This material is based upon work supported in part by the National Science Foundation under Grant No. 1066293 and the hospitality of the Aspen Center for Physics. 


\section{A Self-energy form factors}

Throughout this work the following form factors appear:

$$
\begin{aligned}
f\left(p^{2}, m^{2}, M^{2}\right)= & \frac{1}{576 \pi^{2} m^{2} M^{2}}\left[3\left(2 p^{2}\left(m^{2}+M^{2}\right)-m^{4}-14 m^{2} M^{2}-9 M^{4}\right) A_{0}\left(M^{2}\right)\right. \\
+ & 3\left(2 p^{2}\left(m^{2}+M^{2}\right)-9 m^{4}-14 m^{2} M^{2}-M^{4}\right) A_{0}\left(m^{2}\right) \\
+ & 3\left(24 m^{2} M^{2}\left(m^{2}+M^{2}\right)+p^{2}\left(3 m^{4}-2 m^{2} M^{2}+3 M^{4}\right)\right. \\
& \left.-2 p^{4}\left(m^{2}+M^{2}\right)\right) B_{0}\left(p^{2}, m^{2}, M^{2}\right) \\
+ & 2\left(3\left(m^{6}+11 m^{4} M^{2}+11 m^{2} M^{4}+M^{6}\right)-4 p^{2}\left(m^{4}+m^{2} M^{2}+M^{4}\right)\right. \\
& \left.+p^{4}\left(m^{2}+M^{2}\right)\right) \\
+ & \left.3 \frac{\left(m^{2}-M^{2}\right)^{2}}{p^{2}}\left(m^{4}+10 m^{2} M^{2}+M^{4}\right)\left(B_{0}\left(0, m^{2}, M^{2}\right)-B_{0}\left(p^{2}, m^{2}, M^{2}\right)\right)\right], \\
g\left(p^{2}, m^{2}, M^{2}\right)= & \frac{m^{2}+M^{2}}{32 \pi^{2} m^{2} M^{2}}\left[\left(2 p^{2}\left(m^{2}+M^{2}\right)-p^{4}-\left(m^{2}-M^{2}\right)^{2}\right) B_{0}\left(p^{2}, m^{2}, M^{2}\right)\right. \\
+ & \left.\left(m^{2}-M^{2}+p^{2}\right) A_{0}\left(m^{2}\right)+\left(M^{2}-m^{2}+p^{2}\right) A_{0}\left(M^{2}\right)\right], \\
h\left(p^{2}, m^{2}, M^{2}\right)= & \frac{1}{576 \pi^{2} m^{2} M^{2}}\left[6\left(m^{2}-M^{2}\right)^{2}\left(m^{2}+M^{2}\right)\left(B_{0}\left(0, m^{2}, M^{2}\right)-B_{0}\left(p^{2}, m^{2}, M^{2}\right)\right)\right. \\
+ & 3 p^{2}\left(\left(3 m^{4}+10 m^{2} M^{2}+3 M^{4}\right) B_{0}\left(p^{2}, m^{2}, M^{2}\right)+\left(M^{2}-9 m^{2}\right) A_{0}\left(m^{2}\right)\right. \\
& \left.+\left(m^{2}-9 M^{2}\right) A_{0}\left(M^{2}\right)-2\left(m^{2}+M^{2}\right)^{2}\right) \\
& \left.+p^{4}\left(3\left(A_{0}\left(m^{2}\right)+A_{0}\left(M^{2}\right)\right)+8\left(m^{2}+M^{2}\right)\right)-p^{6}\left(3 B_{0}\left(p^{2}, m^{2}, M^{2}\right)+2\right)\right] .
\end{aligned}
$$

The above expressions simplify when the particles in the loop have the same mass. We define $f\left(p^{2}, m^{2}\right) \equiv f\left(p^{2}, m^{2}, m^{2}\right)$ as the form factor when the masses are equal.

$$
\begin{aligned}
& f\left(p^{2}, m^{2}\right)=\frac{1}{144 \pi^{2} m^{2}} {\left[3\left(12 m^{4}+m^{2} p^{2}-p^{4}\right) B_{0}\left(p^{2}, m^{2}, m^{2}\right)\right.} \\
&\left.+6\left(p^{2}-6 m^{2}\right) A_{0}\left(m^{2}\right)+36 m^{4}-6 m^{2} p^{2}+p^{4}\right], \\
& g\left(p^{2}, m^{2}\right)=\frac{p^{2}}{16 \pi^{2} m^{2}}[\left.\left(4 m^{2}-p^{2}\right) B_{0}\left(p^{2}, m^{2}, m^{2}\right)+2 A_{0}\left(m^{2}\right)\right], \\
& h\left(p^{2}, m^{2}\right)=\frac{p^{2}}{576 \pi^{2} m^{4}}[ 3\left(16 m^{4}-p^{4}\right) B_{0}\left(p^{2}, m^{2}, m^{2}\right)+6\left(p^{2}-8 m^{2}\right) A_{0}\left(m^{2}\right) \\
&\left.-2\left(12 m^{4}-8 m^{2} p^{2}+p^{4}\right)\right] .
\end{aligned}
$$

Notice that when the masses in the above form factors are equal, the form factor vanishes at zero-momentum. This ensures that gauge invariance, which requires $\Pi_{A A}(0)=\Pi_{Z A}(0)=$ 0 , is satisfied. It also ensures that the STUVWX parameters have the correct limiting forms. Consider $S$ in model VII, for example: since the Taylor expansion of any form factor about $p^{2}=0$ starts at one derivative one sees from eqs. (2.7) that the one derivative contribution to the $S$ parameter vanishes. This is precisely as expected from the PeskinTakeuchi definition of S, proportional to $\Pi_{3 B}^{\prime}(0)$ : in model VII the vector boson couples to $B$ but not to $W^{3}$. 
The one-loop form factors have been written in terms of Passarino-Veltman functions

$$
\begin{aligned}
A_{0}\left(m^{2}\right) & =16 \pi^{2} \mu^{4-D} \int \frac{d^{D} q}{i(2 \pi)^{D}} \frac{1}{q^{2}-m^{2}+i \epsilon}, \\
B_{0}\left(p^{2}, m^{2}, M^{2}\right) & =16 \pi^{2} \mu^{4-D} \int \frac{d^{D} q}{i(2 \pi)^{D}} \frac{1}{\left[q^{2}-m^{2}+i \epsilon\right]\left[(q+p)^{2}-M^{2}+i \epsilon\right]} .
\end{aligned}
$$

Open Access. This article is distributed under the terms of the Creative Commons Attribution Noncommercial License which permits any noncommercial use, distribution, and reproduction in any medium, provided the original author(s) and source are credited.

\section{References}

[1] Z. Ligeti, M. Papucci, G. Perez and J. Zupan, Implication $S$ of the dimuon CP asymmetry in $B_{d, s}$ decays, Phys. Rev. Lett. 105 (2010) 131601 [arXiv:1006.0432] [INSPIRE].

[2] A. Lenz, U. Nierste, J. Charles, S. Descotes-Genon, A. Jantsch, et al., Anatomy of new physics in $B-\bar{B}$ mixing, Phys. Rev. D 83 (2011) 036004 [arXiv: 1008.1593] [INSPIRE].

[3] G. Raven, B physics results from LHC, talk presented at Lepton-Photon, August 27 (2011).

[4] D0 collaboration, V.M. Abazov et al., Measurement of the CP-violating phase $\phi_{s}^{J / \psi \phi}$ using the flavor-tagged decay $B_{s}^{0} \rightarrow J / \psi \phi$ in $8 f^{-1}$ of p p collisions, arXiv:1109.3166 [INSPIRE].

[5] R. Chivukula and H. Georgi, Composite technicolor standard model, Phys. Lett. B 188 (1987) 99 [INSPIRE].

[6] L. Hall and L. Randall, Weak scale effective supersymmetry, Phys. Rev. Lett. 65 (1990) 2939 [INSPIRE].

[7] G. D'Ambrosio, G. Giudice, G. Isidori and A. Strumia, Minimal flavor violation: an effective field theory approach, Nucl. Phys. B 645 (2002) 155 [hep-ph/0207036] [INSPIRE].

[8] K. Agashe, M. Papucci, G. Perez and D. Pirjol, Next to minimal flavor violation, hep-ph/0509117 [INSPIRE].

[9] A.V. Manohar and M.B. Wise, Flavor changing neutral currents, an extended scalar sector and the Higgs production rate at the CERN LHC, Phys. Rev. D 74 (2006) 035009 [hep-ph/0606172] [INSPIRE].

[10] J.M. Arnold, M. Pospelov, M. Trott and M.B. Wise, Scalar representations and minimal flavor violation, JHEP 01 (2010) 073 [arXiv:0911.2225] [INSPIRE].

[11] B. Grinstein, A.L. Kagan, J. Zupan and M. Trott, Flavor symmetric sectors and collider physics, JHEP 10 (2011) 072 [arXiv:1108.4027] [INSPIRE].

[12] CDF collaboration, T. Aaltonen et al., Forward-backward asymmetry in top quark production in p p collisions at $\sqrt{s}=1.96$ TeV, Phys. Rev. Lett. 101 (2008) 202001 [arXiv: 0806.2472] [INSPIRE].

[13] D0 collaboration, V. Abazov et al., First measurement of the forward-backward charge asymmetry in top quark pair production, Phys. Rev. Lett. 100 (2008) 142002 [arXiv: 0712.0851] [INSPIRE].

[14] B. Grinstein, A.L. Kagan, M. Trott and J. Zupan, Forward-backward asymmetry in tett $\bar{t}$ production from flavour symmetries, Phys. Rev. Lett. 107 (2011) 012002 [arXiv:1102.3374] [INSPIRE]. 
[15] D0 collaboration, V.M. Abazov et al., Evidence for an anomalous like-sign dimuon charge asymmetry, Phys. Rev. D 82 (2010) 032001 [arXiv: 1005.2757] [INSPIRE].

[16] M. Mulders, Measurement of the top quark mass and the top-antitop invariant mass in $p p$ collisions at $7 \mathrm{TeV}$ with the CMS detector, talk presented at EPS, Grenoble France, July 27 (2011).

[17] M.E. Peskin and T. Takeuchi, Estimation of oblique electroweak corrections, Phys. Rev. D 46 (1992) 381 [INSPIRE].

[18] M. Golden and L. Randall, Radiative corrections to electroweak parameters in technicolor theories, Nucl. Phys. B 361 (1991) 3 [INSPIRE].

[19] B. Holdom and J. Terning, Large corrections to electroweak parameters in technicolor theories, Phys. Lett. B 247 (1990) 88 [InSPIRE].

[20] I. Maksymyk, C. Burgess and D. London, Beyond S, T and U, Phys. Rev. D 50 (1994) 529 [hep-ph/9306267] [INSPIRE].

[21] F. del Aguila, J. de Blas and M. Pérez-Victoria, Electroweak limits on general new vector bosons, JHEP 09 (2010) 033 [arXiv: 1005.3998] [INSPIRE].

[22] B. Grinstein and M.B. Wise, Operator analysis for precision electroweak physics, Phys. Lett. B 265 (1991) 326 [INSPIRE].

[23] C. Burgess, M. Trott and S. Zuberi, Light octet scalars, a heavy Higgs and minimal flavour violation, JHEP 09 (2009) 082 [arXiv: 0907.2696] [INSPIRE].

[24] Particle Data Group collaboration, C. Amsler et al., Review of particle physics, Phys. Lett. B 667 (2008) 1 [inSPIRE].

[25] F. Chen, J.M. Cline and A.R. Frey, Nonabelian dark matter: models and constraints, Phys. Rev. D 80 (2009) 083516 [arXiv:0907.4746] [INSPIRE].

[26] J. Heeck and W. Rodejohann, Kinetic and mass mixing with three abelian groups, Phys. Lett. B 705 (2011) 369 [arXiv: 1109.1508] [INSPIRE]. 